\title{
Maintenance of responding when reinforcement becomes delayed
}

\author{
DANiel S. J. COSTA ANd Robert A. BoAkes \\ University of Sydney, Sydney, Australia
}

\begin{abstract}
In four experiments with rats, we examined the persistence of behavior when reinforcement was switched from immediate to delayed. In Experiment 1, lever pressing elicited by instrumental training with immediate reinforcement continued when a 20 -sec delay of reinforcement was introduced (easy-to-hard condition), whereas when the delay condition was introduced from the start (hard-to-hard condition), responding remained low throughout. A similar result was obtained in Experiment 2, in which lever pressing was elicited by a classical conditioning (autoshaping) procedure. In Experiment 3, rats initially trained with delayed reinforcement continued to respond at a low rate when switched to immediate reinforcement (hard-to-easy condition). By measuring magazine entry (goal tracking) as well as lever pressing (sign tracking) in Experiment 4, we confirmed that such transfer effects at least partly involve the persistence of whatever type of behavior was initially dominant.
\end{abstract}

An important theoretical challenge that has received relatively little attention in recent years is to explain the maintenance of behavior after initial acquisition conditions have been degraded. As pointed out by Rescorla (1989), current associative theories in general predict that if the conditions required to support acquisition of some conditioned behavior are altered deleteriously, this change should produce a corresponding reduction in responding. Most such theories are unable to account for examples in which responding persists, despite a change in the conditions that do not support acquisition of the response. Such an example was provided by Rescorla: He used a classical conditioning procedure with a 1.0 contingency between keylight and food to establish autoshaped key pecking by pigeons, and then he degraded the keylight-food contingency by introducing unsignaled food deliveries. Control subjects exposed to this new contingency from the outset failed to acquire the key-pecking response. In contrast, the pigeons that had initially been trained on the 1.0 contingency continued to peck the keylight under the new conditions with very little change in their response rate.

The particular example examined in the present experiments is the transition from immediate to delayed reinforcement. Since Thorndike (1911), the interval between a response and reinforcement has been recognized as one of the most fundamental factors affecting both the acquisition and maintenance of instrumental behavior. Thus, in the present case of rats pressing a lever that produces a delivery of food, if there is a delay of several seconds between a leverpress and the delivery of food, acquisition of lever pressing is severely retarded, and responding continues at a lower rate than when reinforcement is immediate (see, e.g., Dickinson, Watt, \& Griffiths, 1992;
Logan, 1952; Perin, 1943; Williams, 1999). The empirical question addressed here is what happens when first a rat is trained with immediate reinforcement and then reinforcement is delayed.

Data bearing on this question were obtained by Harker (1956). Three of Harker's five groups are of particular interest here. For one of them (Group II), there was a 10-sec delay between a leverpress and the subsequent delivery of a food pellet throughout 90 trials of training; as expected, this resulted in much slower acquisition than occurred in a group with a 1-sec delay of reinforcement throughout (Group I). The most interesting group (Group III) had a 1 -sec delay of reinforcement for the first 40 trials and was then switched to a 10 -sec delay for the remaining 50 trials. This switch produced no detectable change in performance; rats in this group continued to respond as rapidly as those in the 1-sec group (Group I).

A feature of Harker's (1956) procedure limits the usefulness of his data with respect to our present concerns. He arranged that if a rat had not pressed the lever within $30 \mathrm{sec}$ of the latter's insertion into the chamber, a food pellet would be delivered. Thus, to use much later terminology, he used a procedure similar to autoshaping that combined both classical and instrumental contingencies (Myer \& Hull, 1974). In what appears to be the only published follow-up to Harker's experiment, touching a retractable lever was the instrumental response in an experiment that, like Harker's study, had a discrete-trial procedure to study transitions from short to longer delays of reinforcement. Messing, Kleven, and Sparber (1986) described their procedure as "autoshaping," since if no lever touch had occurred by the end of a 15-sec trial, a response-independent food pellet was delivered. Although in their study the re-

D. Costa, danielc@psych.usyd.edu.au 
inforcement delay lengthened in 2-sec steps-from 0 to 8 sec-instead of the abrupt jump from a 1- to a 10-sec reinforcement delay for Harker's Group III, the main conclusion was the same: Rats that had acquired a response under short delay conditions later continued to respond at a high rate, despite a delay of reinforcement that, if it had been introduced from the outset of training, would have produced only very slow acquisition and only a low level of performance after many training sessions.

The use of a combination of instrumental and classical procedures in these two early experiments makes it difficult to analyze the basis of the transitions from a short to a long delay of reinforcement. In the experiments reported here, we used either a purely instrumental procedure (Experiment 1) or a purely classical procedure (Experiments 2, 3, and 4). In terms of classical conditioning research, a few studies have noted the persistence of human eyeblink responses (Prokasy, Ebel, \& Thompson, 1963) and rabbit nictitating membrane responses (Prokasy \& Papsdorf, 1965) when the interstimulus interval (ISI) was lengthened, either abruptly or in steps, from an initial (and optimal) setting of $500 \mathrm{msec}$ to a value of $2,500 \mathrm{msec}$, which would not support initial acquisition of the conditioned response. More limited easy-to-hard transfer of this kind has been found when the conditioned stimulus modality was also changed as the ISI lengthened (Kehoe \& Holt, 1984). Finally, conditioning an aversion to one taste using a short delay can facilitate subsequent acquisition of long-delay aversion learning to a second taste (Westbrook \& Homewood, 1982).

Our original reason for examining the transition from immediate to delayed reinforcement came from the consideration that it might be analogous to the easy-to-hard effect found in discrimination training, an effect amply documented since Pavlov (1927; see, e.g., Lawrence, 1952). An animal trained from the outset to discriminate between a pair of very similar stimuli (hard condition) may continue to perform poorly despite extensive training, whereas one trained initially to discriminate between a pair of stimuli that are more widely spaced along the same continuum (easy condition) and then transferred to the hard discrimination may show good performance on the latter.

An explanation for such easy-to-hard transfer is offered by Mackintosh's (1975) attentional theory of associative learning and its analysis of transfer along a continuum (see, e.g., Mackintosh \& Little, 1970; Scahill \& Mackintosh, 2004). A possible application of such a theory to transfer from immediate to delayed instrumental reinforcement is to assume that immediate reinforcement increases the associability of the instrumental response and that this increased associability is sustained after the transition to delayed reinforcement, so that the response-reinforcement association remains relatively unaffected. In contrast, when reinforcement is delayed from the outset, the leverpress has to compete with various contextual stimuli for association with reinforcement (Williams, 1999), so its associability is not greatly increased. A closely related possibility follows from the finding that discrimination learning with delayed reinforcement is enhanced if both response alternatives are "marked" (Lieberman, McIntosh, \& Thomas, 1979; Lieberman \& Thomas, 1986). Thus, in the present case, immediate reinforcement may "mark" the single instrumental response in a way that allows the response-reinforcer association to be maintained when the reinforcer is delayed.

A very different account is based on the possibility of competition at a behavioral level. Thus, in Harker's (1956) experiment, although he did not report these data, the group given 10-sec-delayed reinforcement from the outset are likely to have responded on fewer of the trials, and thus to have received more free reinforcements than the group given 1-sec reinforcement from the outset. This would make it possible for free food delivery to reinforce behavior other than lever pressing, and this other behavior would have competed with the lever pressing. Also, given what was discovered later about pairing lever-related stimuli with food delivery, it is likely that the classical contingency would have generated competing sign- and goal-tracking responses (see, e.g., Boakes, 1977); consistent with this suggestion is Harker's note that his rats "frequently took the bar in their teeth or dug in the food cup" (p. 309). On the other hand, by the time that the subjects in Harker's critical group (III) were transferred from 1- to 10 -sec reinforcement, lever pressing would have become their dominant behavior.

A similar explanation could apply to the rats in Messing et al. (1986), which were given an 8-sec delay of reinforcement throughout training. In this case, data were reported showing that at least half of the 12 reinforcers provided in each session followed behavior other than lever touching. What form this behavior took is not known, other than that it was not rearing; the latter was the only other response measured in this study, and it turned out to be unaffected by delay of reinforcement.

Aside from its theoretical interest, transfer from immediate to delayed reinforcement has broad practical implications, particularly in the areas of animal (see, e.g., McGreevy \& Boakes, 2007) and human (e.g., Schmidt $\&$ Bjork, 1992) training. Greater understanding of such transfer in animals may well bring practical benefits.

\section{EXPERIMENT 1}

This experiment simply examined the transition from immediate reinforcement to 20 -sec delay of reinforcement, using a purely instrumental reinforcement contingency in a discrete-trial procedure. It included two groups. In the first stage, group easy-to-hard $(\mathrm{EH})$ was trained to press a lever with immediate reinforcement, and in the second stage, reinforcement was delayed by $20 \mathrm{sec}$. The control group, group hard-to-hard $(\mathrm{HH})$, was trained with 20 -sec-delayed reinforcement throughout. The choice of $20 \mathrm{sec}$ was based on preliminary parametric studies (Costa, 2004).

Other differences from Harker (1956) and Messing et al. (1986) included the use in the present experiment of a fixed-interval (FI) schedule during each trial, meaning that only responses performed after a specified interval were reinforced. This allowed the use of rate of respond- 
ing as a measure, whereas the two previous experiments could use as dependent variables only latency on each trial and proportion of trials with a response, since both used continuous reinforcement - that is, the lever was removed and reinforcement was triggered by a rat's first response on the lever. The most important difference between this and the earlier experiments was that, if a rat failed to respond appropriately, no reinforcement occurred on that trial.

\section{Method}

Subjects. Sixteen female hooded Wistar rats from the University of Sydney's psychology breeding program were used in the experiment. They were 160-167 days old at the start of the experiment, with a mean weight of $227 \mathrm{~g}$ (range 211-238 g). The rats had previously participated in a running wheel experiment. They were housed in a colony room in large polyurethane cages measuring $26 \times 59 \times$ $37 \mathrm{~cm}$, with 8 rats per cage. A food deprivation schedule provided 2-h access to food following each daily session, and unrestricted water access was given in the home cages.

Apparatus. Eight conditioning chambers (Skinner boxes), measuring $30 \times 26 \times 31 \mathrm{~cm}$, were housed in sound- and light-resistant shells. The two end walls and ceiling of each chamber were aluminum; the side walls were clear Plexiglas. The floor of each chamber was composed of 16 stainless steel rods, and 45-mg Noyes Precision food pellets (Formula 1) could be delivered into a magazine located in the center of one end wall. The same wall contained two 48-mmwide retractable levers (MED Instruments Inc.), one on either side of the magazine aperture, but only the right-hand lever was used in this experiment. This lever was mounted such that, when extended, the lever projected $19 \mathrm{~mm}$ into the chamber, with its top surface $65 \mathrm{~mm}$ above the floor of the chamber. The distance from the left-hand edge of the lever to the center of the magazine aperture was $65 \mathrm{~mm}$.

Procedure. Animals first received a 5-min habituation session in the chambers, then three sessions of magazine training, each consisting of 20 food pellet deliveries on a variable-time (VT) 30-sec schedule. All animals were trained on the lever response with an initial two sessions of continuous reinforcement - that is, the first response in each trial was reinforced. Then Stage 1 of instrumental training commenced. All animals progressed from two sessions on an FI 6-sec schedule, in which only responses occurring $6 \mathrm{sec}$ after lever insertion were reinforced, then two sessions with a 12 -sec FI, and finally three sessions with an 18-sec FI. A discrete-trial procedure was used with a variable intertrial interval of $60 \mathrm{sec}$ (i.e., VT
$60 \mathrm{sec}$ ); the intertrial interval was defined as the period commencing with a reinforced response or lever retraction on one trial and ending with lever insertion for the next trial. Each session contained 20 trials. A trial began with insertion of the lever and ended after $30 \mathrm{sec}$, with retraction of the lever if no response had been made at the appropriate time (i.e., within a time frame set by the current FI schedule). When a rat responded within this time, the lever was immediately retracted, and reinforcement was delivered after a delay that varied across stages. At the beginning of every session (one per day), an animal was placed in its chamber with the lever retracted and the houselight illuminated for $10 \mathrm{~min}$ before training started (see Dickinson et al., 1992).

Instrumental training was divided into two stages. Stage 1 consisted of seven sessions, in which a response made at the appropriate time by subjects in Group EH was immediately reinforced, but responding at the appropriate time was reinforced for Group $\mathrm{HH}$ only after a 20 -sec delay. In Stage 2, which commenced the day after the end of Stage 1 and consisted of six sessions, for all animals an appropriate response at FI $18 \mathrm{sec}$ was reinforced after a delay of $20 \mathrm{sec}$.

\section{Results}

As can be seen in Figure 1, Group EH responded at a much higher rate than Group HH throughout Stages 1 and 2 . The change from immediate to 20 -sec-delayed reinforcement produced an immediate decrease of response rate in Group EH, but within two sessions the rate began to return to a much higher level. In contrast, responding by Group HH occurred at a low rate throughout.

Differences between the two groups were analyzed using Mann-Whitney tests, since the data were far from normally distributed. Using a predefined criterion for the acquisition of lever pressing of 8 out of 10 successive trials with a response (resp.), the median number of trials to criterion in Group EH was 37.5 (i.e., within the first two sessions, in which all rats reached the criterion), but no subject in Group HH reached this criterion. On the final day of Stage 1, the median response rate in Group EH (50.4 resps./min) was significantly greater than the median rate in Group HH $(2.4$ resps. $/ \mathrm{min})[U(8,8)=0$, $p<.05]$. On the final day of Stage 2 , the median response rate in Group EH (43.2 resps./min) was still significantly

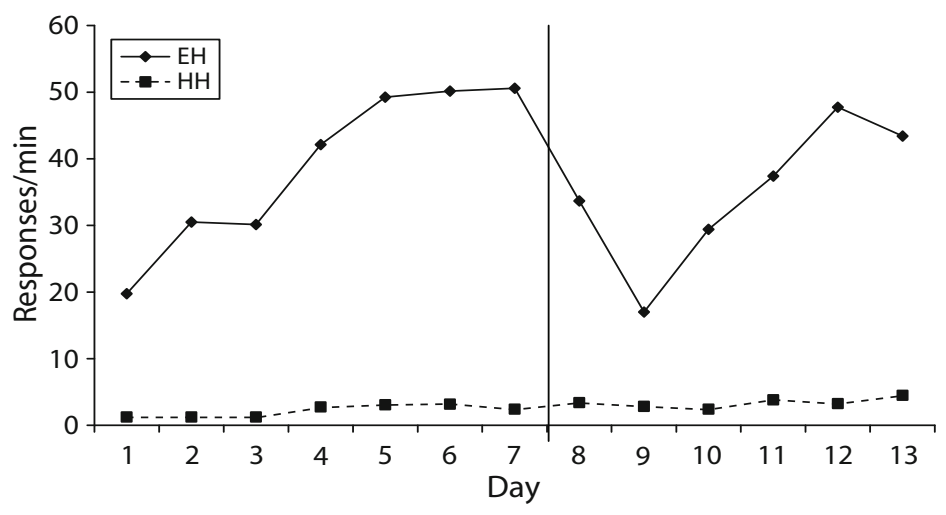

Figure 1. Experiment 1: Median response rates for Groups EH and HH over Stages 1 and 2. Subjects in Group EH were transferred from immediate reinforcement to delayed reinforcement on Day 8 (the vertical line indicates the transition from Stage 1 to Stage 2). An instrumental procedure was used with food pellets as the reinforcer. 
greater than the median in Group HH (4.5 resps./min) $[U(8,8)=2, p<.05]$, even though the two groups had been given an identical reinforcement schedule over the final six sessions.

The low rate of responding in Group HH may be related to the fact that on many of the trials these rats failed to make a criterion response, and thus did not receive a food pellet. Thus, in the final session of Stage 1, Group EH received a median of 20 out of 20 possible pellets, whereas Group HH received a median of only 6 pellets in this ses$\operatorname{sion}[U(8,8)=0, p<.05]$.

\section{Discussion}

The overall pattern of results was more dramatic than those reported by Harker (1956) and Messing et al. (1986), in that here initial instrumental training with immediate reinforcement produced persistent responding under later conditions of 20-sec-delayed reinforcement that were hardly adequate to support acquisition of the response. Harker suggested that the secondary reinforcing effect of lever retraction following a response might be responsible for continued responding after the switch to delayed primary reinforcement in his Group III. This explanation seems less likely for the present pattern of responding in Group EH in that, unlike in Harker's Group III, responding decreased quite sharply in the two sessions following introduction of a 20-sec delay in reinforcement. Had retraction of the lever become a strong conditioned reinforcer, responding should have been little affected by the change, as observed by Harker when his Group III was switched from a 1 -sec to a 10 -sec delay. In addition, it seems unlikely that responding could have recovered from the initial decrease, given that lever retraction was no longer immediately paired with food, so its effectiveness as a secondary reinforcer should have weakened. Instead, the decline and then recovery of responding shown by Group $\mathrm{EH}$ is consistent with the proposal that in this group the response retains the high associability it acquired in Stage 1, thus allowing acquisition of an association between the response and the delayed reinforcer in Stage 2.

An alternative explanation for the different response rates in Groups $\mathrm{EH}$ and $\mathrm{HH}$ arises from a consideration of reinforcement rates. In Stage 1, Group EH presumably came to associate the conditioning chamber with a higher rate of reinforcement than did Group HH. Other things being equal, contexts associated by hungry animals with high rates of reinforcement tend to produce higher levels of activity (see, e.g., Rescorla, Durlach, \& Grau, 1985). Thus, the more often Group EH made the required leverpress, the higher the level of activity and, thus, the higher the likelihood of responding appropriately on the next trial. In contrast, Group HH might have suffered from a vicious circle effect: The less they responded, the lower the reinforcement rate and, as a consequence, the lower their activity level. This is a challenge when shaping new behavior (see, e.g., Midgley, Lea, \& Kirby, 1989; Pear \& Legris, 1987). Such a vicious circle is not a problem when interpreting Harker's (1956) results, since his procedure ensured that all of his rats received a food pellet on every trial. Although it avoided the difficulties of interpretation, his procedure of giving a free pellet at the end of a trial in which a rat failed to respond created the other problems that we noted earlier.

\section{EXPERIMENTS 2A AND 2B}

To overcome the problem of differential reinforcement rates inherent in the kind of purely instrumental conditioning procedure employed in Experiment 1, these experiments examined the effect of a transition from immediate to delayed reinforcement using a purely classical conditioning procedure, in which the delivery of reinforcement was entirely independent of a rat's behavior. The procedure was based on insertion of the lever for a fixed time as the conditioned stimulus (CS) and delivery of either a pellet (Experiment 2A) or sucrose (Experiment 2B) as the unconditioned stimulus (US). As is widely documented (see, e.g., Myer \& Hull, 1974), such a procedure can produce sign-tracking behavior taking the form of lever pressing, as recorded in the present experiments, instead of just lever contacts, as recorded by Messing et al. (1986; cf. Stiers \& Silberberg, 1974). The standard convention in classical conditioning for referring to an unfilled interval separating the end of a CS and the onset of the US is to use the term trace interval; however, for consistency with the use in instrumental conditioning, it will be referred to here as the delay of reinforcement, as in Experiment 1.

The basic design of both experiments was the same as that of Experiment 1. During an initial stage of training, for Group EH the occasional insertion of a lever for $10 \mathrm{sec}$ was followed by reinforcement immediately after retraction, but in the second stage reinforcement was delayed for $10 \mathrm{sec}$ after the lever had been retracted on each trial. For Group HH, a reinforcement delay of $10 \mathrm{sec}$ was used throughout training. The choice of a $10-\mathrm{sec}$ delay was based on extrapolating from Messing et al. (1986) a delay likely to support little lever pressing, if introduced from the outset, but likely to sustain responding in Group EH.

The only difference between Experiments 2A and 2B was the use of food pellets in the first and $20 \%$ sucrose in the second of these experiments as the reinforcer. The switch to sucrose was made because some of the pellet dispensers had become unreliable; sucrose also had the advantage of allowing a check that the basic phenomenon was not reinforcer specific.

\section{Method}

Subjects and Apparatus. In Experiment 2A, the subjects were 32 female Wistar rats 94-128 days old, with a mean weight of $208.1 \mathrm{~g}$ (range 149-261 g) at the start of the experiment. Of these rats, 24 were hooded and naive, and 8 were albinos that had previously participated in running wheel experiments. Allocation to the two groups was equated across the two kinds of rat. All animals were housed in the colony room in groups of 8. The reinforcers in Experiment 2A were 45-mg Noyes Precision food pellets (Formula 1), as in Experiment 1.

In Experiment 2B, 16 naive female hooded Wistar rats were used; they were 118 days old, with a mean weight of $194.6 \mathrm{~g}$ (range 182$206 \mathrm{~g}$ ) at the start of the experiment. These animals were also housed in groups of 8 . Reinforcement was $20 \%$ sucrose mixed in tap water, delivered from $0.1-\mathrm{ml}$ dipper cups via a dipper raised for $5 \mathrm{sec}$. 
In both experiments, the animals were maintained on a food deprivation schedule with 2 -h access to food in the home cages following each daily experimental session. The apparatus used was the same as in Experiment 1, except that fluid dippers (MED Associates) were used in Experiment 2B.

Procedure. In Experiment 2A, magazine training with food pellets was performed exactly as in the previous experiment. In Experiment 2B, magazine training consisted of one session of 30 sucrose deliveries on a VT 60 -sec schedule, with dipper time set at $7 \mathrm{sec}$, followed by a second session of 20 sucrose deliveries on a VT 60 -sec schedule and a dipper time of $6 \mathrm{sec}$. In the subsequent two stages of classical conditioning, each session contained 20 trials on a VT 60 -sec schedule. Each trial commenced with a $10-$ sec insertion of the lever and concluded with the delivery of a reinforcer either immediately or after $10 \mathrm{sec}$, depending on the stage and group. In the five sessions of Stage 1, a reinforcer was delivered immediately after lever retraction for Group EH but only after a further $10 \mathrm{sec}$ for Group HH. In the five sessions of Stage 2, reinforcer delivery was delayed for $10 \mathrm{sec}$ in both groups.

\section{Results and Discussion}

As shown in Figure 2, in the first stage of both experiments Group EH pressed the lever at a steadily increasing rate that was hardly affected by the switch to a 10 -sec delay of reinforcement in the second stage. In contrast, in both experiments Group HH responded at the same low rate over the course of Stages 1 and 2.

In Experiment 2A, 12 of the 16 rats in Group EH had reached the predefined criterion of 8 trials out of 10 with a response by the end of Stage 1, whereas only 1 of the 16 rats in Group HH achieved the criterion $\left[\chi^{2}(1)=15.68\right.$, $p<.05]$. By the end of the experiment, 15 of the 16 rats in Group EH had reached the criterion, whereas only 3 of the 16 rats in Group HH did so [ $\left.\chi^{2}(1)=18.29, p<.05\right]$. In the final session of the first stage (Day 5), response rates in Group EH (median 7.8 resps./min) were significantly higher than those in Group HH (median 1.2 resps./min) [Mann-Whitney $U(16,16)=23, p<.05]$. More importantly, in the final session of the second stage (Day 10), response rates in Group EH (median 11.4 resps./min) were still significantly higher than those in Group $\mathrm{HH}$ (median $1.2 \mathrm{resps} . / \mathrm{min})[U(16,16)=42, p<.05]$.

In Experiment 2B, 6 of the 8 rats in Group EH had reached the predefined criterion of 8 trials out of 10 with a response by the end of the experiment, whereas none of the 8 rats in Group HH had done so $\left[\chi^{2}(1)=9.6, p<\right.$ $.05]$. On Day 5, response rates in Group EH (median $13.4 \mathrm{resps} . / \mathrm{min}$ ) were higher than those in Group HH
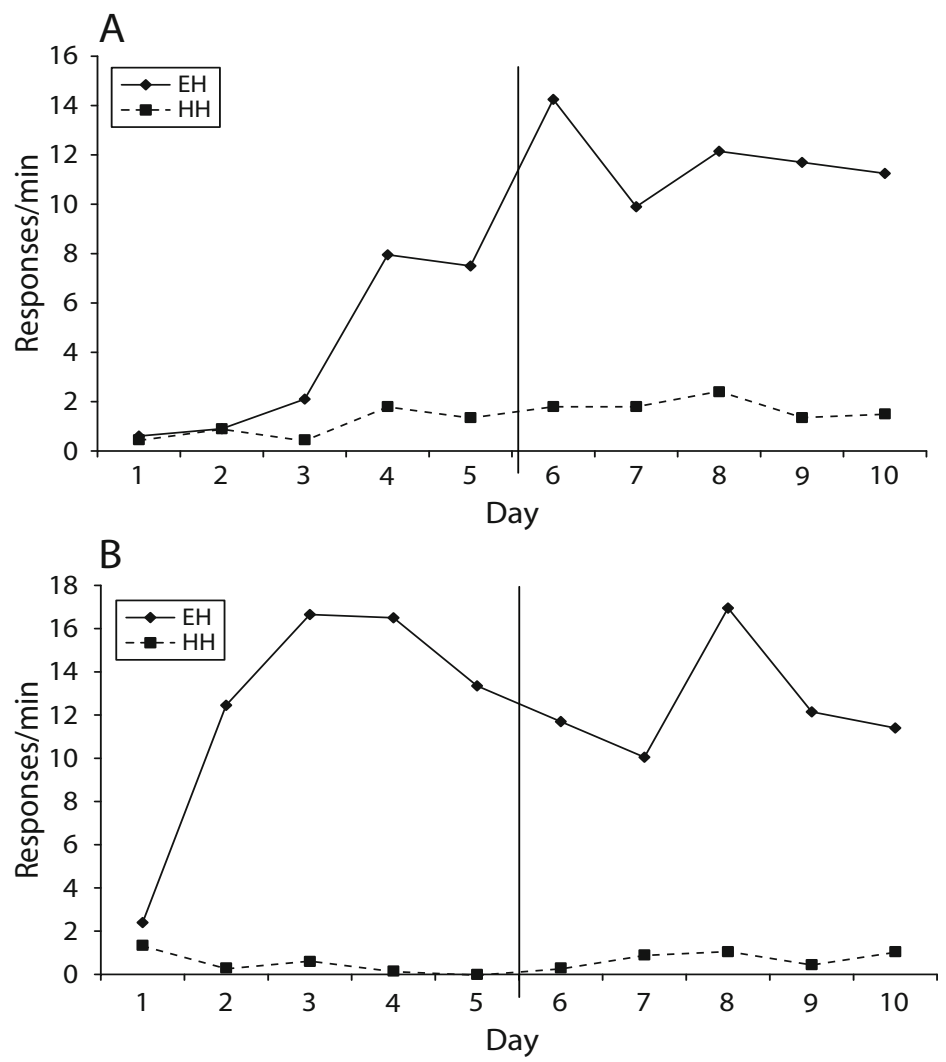

Figure 2. (A) Experiment 2A: Median response rates for Groups EH and HH over Stages 1 and 2 , with food pellets used as the reinforcer. (B) Experiment 2B: Median response rates for Groups EH and HH over Stages 1 and 2, with sucrose used as the reinforcer. In both experiments, a classical conditioning procedure was employed and subjects in Group EH were transferred from immediate reinforcement to delayed reinforcement on Day 6. 
(median 0 resps./min), a marginally significant result $[U(8,8)=13, p=.05]$. This difference continued into the second stage, so that in the final session (Day 10) response rates in Group EH (median 11.4 resps./min) were still significantly higher than those in Group HH (median $1.2 \mathrm{resps} . / \mathrm{min})[U(8,8)=8, p<.05]$. As with the similar result in Experiment 2A, this difference confirms transfer from immediate to 10 -sec-delayed reinforcement.

\section{EXPERIMENT 3}

The previous experiments served to demonstrate that our type of easy-to-hard transfer can occur with both purely instrumental and purely classical procedures. However, the experiments did not provide much guidance as to the processes underlying such transfer. As noted earlier, when instrumental conditioning procedures are used in between-groups comparisons, differential reinforcement rates produced by differences in responding between the groups can obscure the possible contribution of other factors to transfer from short to long delay of reinforcement. Consequently, Experiment 3 employed the same classical conditioning procedure used in Experiments $2 \mathrm{~A}$ and $2 \mathrm{~B}$ in order to ensure that reinforcement rates were equated across groups.

The aim of Experiment 3 was to test whether, under the general conditions of Experiment 2B, the retarded acquisition of lever pressing when there was a 10-sec delay between lever retraction and delivery of the reinforcer was due to the development of behavior other than lever pressing. The presence of multiple forms of conditioned responding has been demonstrated in both rats (Holland, 1977) and pigeons (Brown, Hemmes, Cabeza de Vaca, \& Pagano, 1993). According to this theory, rather than failing to associate the lever with the delayed arrival of sucrose, rats acquire the "wrong" behavior. It follows that, if rats are first trained on a long delay and then shifted to immediate reinforcement-Group HE ("hard-to-easy") in the present experiment-this other behavior will interfere with the acquisition of lever pressing. The control condition was one that provided equivalent exposure to both lever insertions and reinforcement deliveries without using uncorrelated exposure to these two events (i.e., a truly random condition) that might produce learned irrelevance (Bonardi \& Ong, 2003). Consequently, the control group, Group Block-E, was given blocked exposure to both events in the first stage, followed in the second stage by the same "easy" condition of immediate reinforcement given to Group HE.

\section{Method}

Subjects and Apparatus. The 16 naive female hooded Wistar rats in this experiment were 109 days old, with a mean weight of $172 \mathrm{~g}$ (range 136-212 g) at the start of the experiment. Housing and access to food and water were exactly as in the previous experiments. The apparatus was the same as in Experiment 2B.

Procedure. For the two groups in this experiment (HE and Block-E), magazine training proceeded as in Experiment 2B. In the first stage, the conditions for Group HE were the same as those for Group $\mathrm{HH}$ in Experiment 2B: Each trial commenced with a 10 -sec insertion of the lever, followed $20 \mathrm{sec}$ later by the delivery of noncontingent sucrose solution, and each of the six sessions contained 20 trials on a VT $60-\mathrm{sec}$ schedule. In this stage, rats in Group Block-E received sessions without sucrose in which the lever was inserted for $10 \mathrm{sec} 40$ times on a VT 60 -sec schedule, as well as sessions without lever insertion in which they received 40 deliveries of sucrose, also on a VT 60 -sec schedule. For the first 3 days, one subgroup was given lever-only sessions, and the second subgroup was given sucrose-only sessions. These conditions were reversed for the final 3 days. In the four sessions of the second stage, conditions were identical for the two groups and consisted of immediate sucrose delivery following retraction of the lever, exactly as in the first stage for Group EH in Experiment 2B.

\section{Results}

As shown in Figure 3, during the first stage, when reinforcement was delayed for $10 \mathrm{sec}$, the results for Group HE were exactly like those for Group HH in Experiment 2B, in that these rats rarely pressed the lever. The important

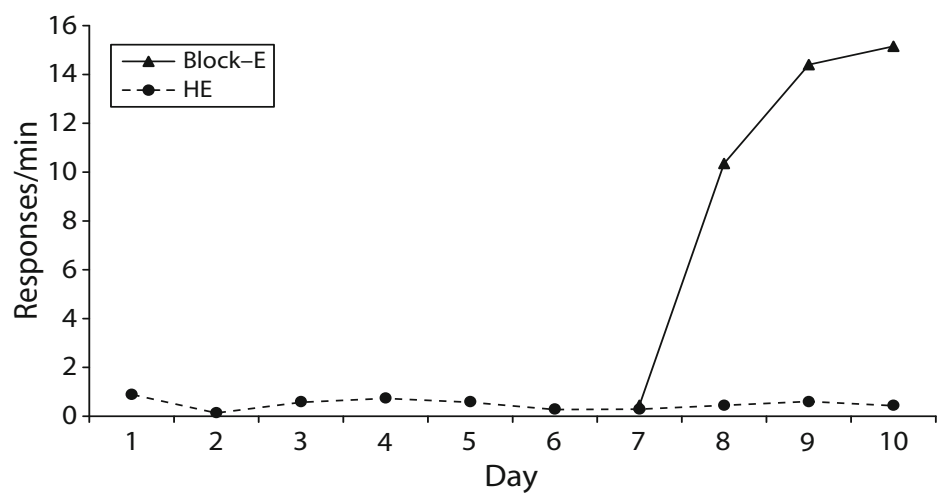

Figure 3. Experiment 3: Median response rates for Groups Block-E and $H E$ over Stages 1 and 2. A classical procedure was used with sucrose as the reinforcer. For the six sessions of Stage 1, Group HE was given a 10-sec delay of reinforcement, and Group Block-E was given blocks of three sessions of sucrose only and three sessions of lever insertion only. In Stage 2, for both groups sucrose followed immediately after lever retraction. 
result was that this group did not respond any more in the second stage, when reinforcement became immediate, whereas Group Block-E showed rapid acquisition of lever pressing; no difference was detected between its two subgroups. The median number of trials to reach the criterion of 8 trials out of 10 with a response was 33 in Group Block-E (with all but 1 of the rats in that group reaching criterion), which was almost identical to the median number for Group EH in Experiment 2B (32.5 trials). Thus, this between-experiments comparison gave no indication that blocked exposure to lever insertion and sucrose delivery retarded acquisition of lever pressing.

Only 2 rats in Group HE reached the acquisition criterion: 1 in the first stage and 1 in the second. Thus, exposure to a $10-\mathrm{sec}$ delay of reinforcement drastically interfered with leverpress acquisition, even when reinforcement became immediate. The difference between the two groups was also seen in terms of response rates in the final session of the second stage: On Day 10, the median response rate of Group Block-E (15.0 resps./min) was significantly higher than that of Group HE $(0.6$ resps. $/ \mathrm{min})[U(8,8)=$ $2, p<.05]$.

\section{Discussion}

The clear outcome of this experiment was that exposure to a 10 -sec delay of reinforcement prevented subsequent acquisition of lever pressing under conditions of immediate reinforcement that would normally-as with Group EH in Experiment 2B and Group Block-E here-produce a steady and moderate rate of responding on the lever. This finding is consistent with the view that the normal absence of lever pressing when reinforcement is delayed for $10 \mathrm{sec}$ reflects the development of other behavior. What this other behavior might be was not systematically analyzed in the present experiment. However, casual observation suggested that rats in the long-delay condition generally responded to insertion of the lever by approaching and staying close to the magazine aperture (i.e., they showed goal-tracking behavior; Boakes, 1977; Brown et al., 1993).

An unexpected feature of these results was the rapid acquisition of lever pressing by Group Block-E. We had expected acquisition by this group to be somewhat slower than that by Group EH in the first stage of Experiment 2B, because the blocked condition seemed likely to produce strong context-sucrose associations that might then interfere with acquisition of lever-sucrose associations, especially in the subgroup given repeated exposure to sucrose just before the second stage (see Reilly, Schachtman, \& Reid, 1996; Williams, 1999). Moreover, prior exposure to lever insertions might have produced a latent inhibition effect that would have slowed acquisition by Group Block-E in the second stage. The experiment was not designed to detect such CS and US preexposure effects, and indeed they may have been present; however, the fact that Group Block-E acquired lever pressing at essentially the same rate as Group EH in Experiment 2B indicates that the influence of preexposure was minor in this case.

\section{EXPERIMENT 4}

Following the informal observation of goal tracking in the previous experiment, the conditioning chambers were modified to allow the recording of magazine entries. Using the same procedures as in Experiments 2 and 3, one aim of the present experiment was to test the prediction that during an initial training period, magazine entries in the presence of the lever would be more frequent in the delay condition than in the immediate condition. Brown et al. (1993) found that when pigeons received food signaled by a keylight, the pigeons tended to approach the keylight when food immediately followed the offset of the keylight, but tended to approach the food delivery area when a trace interval of $6 \mathrm{sec}$ between keylight offset and food was present. Hence, in the present study we predicted that sign tracking, rather than goal tracking, would develop in rats first trained with no delay, whereas the converse would be the case for those initially trained with a 10 -sec delay.

The second aim was to examine whether the dominant behavior during the initial stage would persist when the timing of the reinforcement was changed. To this end, one group $(\mathrm{EH})$ was initially trained with immediate reinforcement and then switched to delayed reinforcement, exactly as the EH groups had been in Experiment 2. For a second group (HE), the order of these conditions was reversed, exactly as with the HE group in Experiment 3. This design allowed us to see, for example, whether the pattern of responding shown by a rat given immediate reinforcement in the second stage was more like the pattern it had shown under delayed reinforcement conditions in the preceding stage than like those shown by rats given immediate reinforcement in the initial stage.

\section{Method}

Subjects. The 16 female hooded Wistar rats in this experiment had previously served in a wheel-running experiment. They were 164 days old, with a mean weight of $237 \mathrm{~g}$ (range 212-272 g) at the start of the present experiment. Housing and access to food and water were exactly as in the previous experiments.

Apparatus. The apparatus used in the previous experiments was modified in several ways. Most important was the installation in all eight conditioning chambers of infrared photocell receptors to record entry into the magazine. The infrared beam was recessed $20 \mathrm{~mm}$ into the magazine.

Procedure. For both groups, magazine training for dipperpresented sucrose solution proceeded as in Experiments $2 \mathrm{~B}$ and 3. In the two conditioning stages, the conditions for Group EH were the same as those for Group EH in Experiment 2B, with six sessions of immediate reinforcement followed by four sessions of 10-sec delay of reinforcement. Likewise, the conditions for Group HE were the same as those for Group HE in Experiment 3, with six sessions of 10 -sec delay of reinforcement followed by four sessions of immediate reinforcement.

\section{Results}

Magazine entries while the lever was inserted are shown in the top panel of Figure 4. This shows CS-pre-CS difference scores - the number of magazine entries during the 10 -sec lever insertion period minus the number of entries 


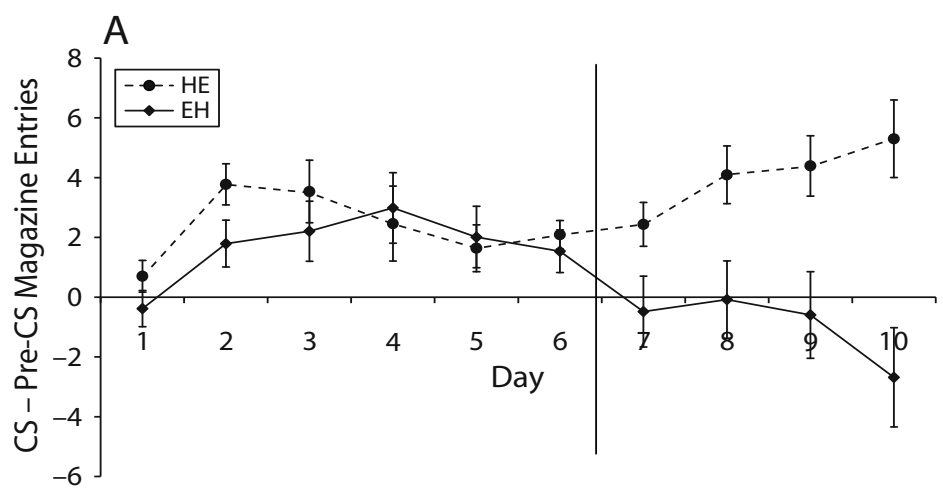

B

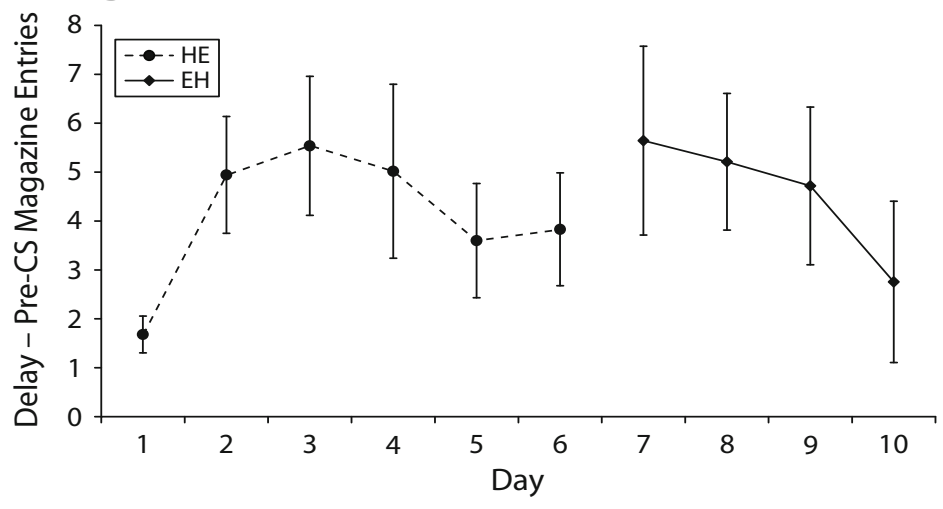

Figure 4. Experiment 4. (A) Mean differences between number of magazine entries during lever insertion (CS) and during the 10-sec (pre-CS) interval prior to lever insertion for Groups HE and EH over the six sessions of Stage 1 and four sessions of Stage 2. A classical procedure was used with sucrose as the reinforcer. (B) Mean differences between number of magazine entries during the delay period and during the 10-sec (preCS) interval prior to lever insertion for Group $\mathrm{HE}$ in Stage 1 and Group EH in Stage 2. In both panels, error bars represent \pm 1 standard error.

in the $10 \mathrm{sec}$ prior to lever insertion - for each day of training. It may be seen that during the initial period of training the two groups did not differ on this measure, but when conditions were reversed magazine entries became more frequent in Group $\mathrm{HE}$ - the rats that were now given immediate reinforcement - but less frequent in Group EH.

Unlike leverpresses, the magazine entry data in this experiment were normally distributed and hence could be analyzed using repeated measures analysis of variance. In Stage 1, this difference score, averaged over the 6 training days, did not differ significantly between Groups $\mathrm{HE}$ $(M=2.37, S D=2.36)$ and $\mathrm{EH}(M=1.69, S D=2.77)$ $[F(1,14)=0.585, p=.457]$. Although trend analysis failed to reveal a significant linear trend $[F(1,14)=1.57$, $p=.231]$, both the quadratic $[F(1,14)=9.45, p=.008]$ and cubic $[F(1,14)=5.01, p=.042]$ trends were significant. None of these trends interacted with group (all $p \mathrm{~s}>$ .10). Thus, contrary to our prediction, there was no evidence at all for a difference between the groups on this measure at this stage.

In Stage 2, the CS-pre-CS difference scores were higher for Group HE than for Group $\mathrm{EH}[F(1,14)=21.50, p<$ $.0005]$. Trend analysis confirmed an interaction between linear trend and group $[F(1,14)=11.12, p=.005]$, but no other effects or interactions were significant [largest $F(1,14)=3.53]$.

When rats were in the delayed reinforcement condition, we also measured the rate of magazine entries during the delay period to obtain a further difference score: the number of magazine entries during the 10-sec delay minus the number of entries in the 10 -sec interval prior to lever insertion. As can be seen in the lower panel of Figure 4, in Stage 1 the rats in Group HE rapidly developed magazine-oriented responding during the delay period, whereas in Stage 2 the initially high rate of responding in Group EH decreased as the session progressed. Trend analysis revealed that for Group HE (Stage 1), the linear trend was not significant $[F(1,7)=1.09, p=.332]$, but the quadratic trend approached significance $[F(1,7)=$ $5.53, p=.051]$. None of the higher order trends were significant $(p s>.10)$. For Group EH (Stage 2), the linear $[F(1,7)=2.51, p=.157]$, quadratic $[F(1,7)=0.98, p=$ $.356]$, and cubic $[F(1,7)=0.356, p=.570]$ trends were not significant.

As in the previous experiments, in Stage 1 lever pressing hardly occurred in the delay condition, as shown in 


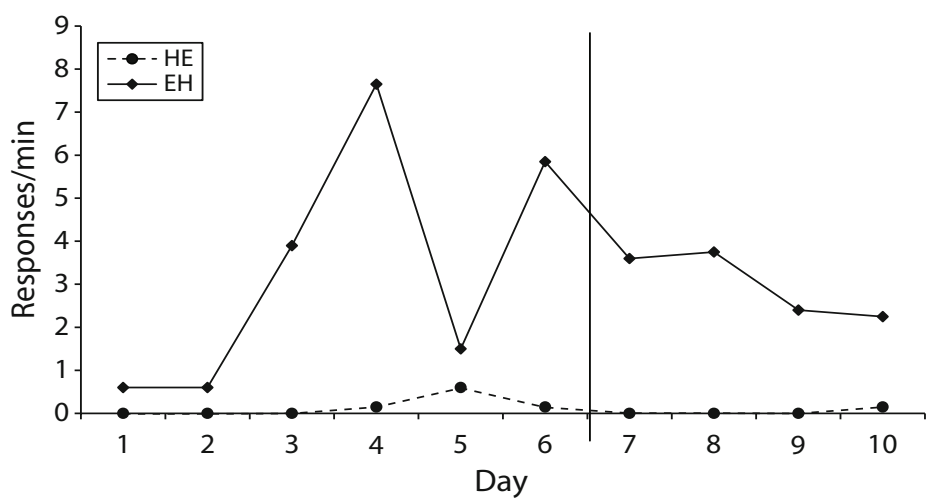

Figure 5. Experiment 4: Median response rates for Groups $\mathrm{HE}$ and EH in Stages 1 and 2.

Figure 5. However, in this case lever pressing was also less consistent in the immediate condition than it had previously been across rats. The median rates for Group EH in the first stage were much lower than for the equivalent groups in Experiment 2B, and as detailed below, several subjects did not produce recordable lever responses. Nevertheless, those rats that did press the lever persisted in doing so when switched from immediate to delayed reinforcement, but their rates declined. On the final day of Stage 1, rats in Group EH (median 5.85 resps./min) were lever pressing at a significantly higher rate than rats in Group HE (median $0.15 \mathrm{resps} . \mathrm{min})[U(8,8)=11, p<.05]$. On the final day of Stage 2, however, there was no significant difference in lever pressing between EH (median 2.25 resps./min) and HE (median 0.15 resps. $/ \mathrm{min})[U(8,8)=20.5, p>.05]$. Throughout the present experiment, only 4 out of 8 rats in Group EH recorded leverpresses with any consistency. Observation revealed, however, that 3 of the 4 other rats in the group regularly made contact with the lever upon its insertion, including 1 rat that vigorously pressed the lever from beneath (which was not recorded).

\section{Discussion}

The most important finding from the magazine entry data in the first stage of this experiment is that on this measure rats learned about the lever-reinforcer contingency as rapidly with a 10 -sec delay as with immediate reinforcement, as can be seen in the upper panel of Figure 4. This indicates that the differences in lever pressing between Groups EH and HH in the first stage of Experiment 2 and between the two groups in the second stage of Experiment 3 were largely differences in performance rather than in associative strength. The rapid increase in magazine entries during the delay period shown in the initial stage by Group HE (see the lower panel of Figure 4) also indicates that the rats learned very rapidly the contingency between lever insertion and the 10-sec-delayed arrival of sucrose.

The lack of any difference in speed of acquisition or maintained rate of magazine responding between the two groups in the present experiment was not what we had expected. When rats were transferred to different delay conditions, however, those in Group HE responded to the magazine at a higher rate than those in Group EH, as predicted. Thus, the HE group in this experiment confirmed the general prediction that the pattern of responding initially acquired to the lever-in this case, goal tracking without sign tracking - would persist despite the shift from delayed to immediate reinforcement. On the other hand, the individual variation seen in Group EH made it more difficult to draw firm conclusions. The most intriguing result from this group was that, although magazine entry during the presence of the lever was established by immediate reinforcement, when the delay was introduced in the second stage this behavior also became quickly delayed, in that now the rats only entered the magazine when the lever was retracted.

The low and inconsistent rate of lever pressing by Group EH remains unexplained. However, given the observation of lever contacts with no leverpresses, the effect illustrated in the recorded data appears to be a conservative reflection of sign tracking.

\section{GENERAL DISCUSSION}

The main findings from these experiments may be summarized as follows. Under the present conditions, in which a lever was inserted into the conditioning chamber every $1 \mathrm{~min}$ on average, most rats with no prior training failed to acquire lever pressing both when reinforcement depended on that response but was delayed by $20 \mathrm{sec}$ (Experiment 1) and when reinforcement was response independent but delayed by $10 \mathrm{sec}$ (Experiments 2, 3, and 4). In contrast, rats first trained with immediate reinforcement that produced acquisition of lever pressing, via either instrumental or Pavlovian conditioned responding, continued to respond under the delayed reinforcement conditions above (Experiments 1 and 2). Such positive "easy-to-hard" transfer contrasted with the negative transfer of lever pressing found in Experiments 3 and 4, in which initial training on delayed reinforcement prevented subsequent acquisition of lever pressing when reinforcement became immediate. 
Experiment 4 revealed that this negative transfer of lever pressing was accompanied by positive transfer of magazine entry behavior.

The positive transfer found here provides a further example to add to findings of transfer along a continuum in discrimination learning (e.g., Scahill \& Mackintosh, 2004) and to the "high-to-low contingency" effect reported by Rescorla (1989); all three effects show that conditions insufficient to produce acquisition of a response can nonetheless maintain responding acquired under more favorable conditions. A further, and very familiar, example is that of positive transfer from continuous instrumental reinforcement to some intermittent schedule of reinforcement, such as a ratio schedule of a value high enough that few subjects will acquire the instrumental response if given this schedule from the outset. That particular example does not seem as problematic as the previous ones listed above, in that positive transfer can be understood in terms of widely accepted principles regarding the development of resistance to extinction following partial reinforcement (Mackintosh, 1974, pp. 434-467; see also the analysis of resistance to change within behavioral momentum theory, e.g., in Nevin \& Grace, 2000). The present kind of easyto-hard transfer is not as easily understood.

In the case of positive transfer from immediate to delayed reinforcement in instrumental conditioning (Experiment 1), one possibility relies on the observation that rates of reinforcement are reduced by delaying reinforcement. As previously discussed, this could lead to maintenance of a low level of activity in Group HH, whereas all that would be needed for Group EH to maintain a high level of responding when delay of reinforcement was introduced would be for the effect of the initial conditions - that is, instrumental responding at a high rate-to persist long enough to maintain their high rate of reinforcement. This explanation remains speculative, in the absence of any measure of activity other than lever pressing. Nonetheless, the differential reinforcement rates in the initial stage make it difficult to assess other explanations for easy-tohard transfer of instrumental responding.

The use of a classical conditioning procedure in Experiments $2 \mathrm{~A}$ and $2 \mathrm{~B}$ ensured that reinforcement rates were then the same throughout for Groups EH and HH. In the introduction, an associative analysis that included an attentional assumption was compared with a nonassociative analysis based on competition at a behavioral level. Assuming that immediate reinforcement increases the effective salience or associability of lever insertion as a CS can account for the positive transfer found in these experiments; such enhanced associability would allow the rat to continue to associate the lever with reinforcement even when the latter was delayed. The implication here is that without the increased associability of the lever, the delay of $10 \mathrm{sec}$ made it difficult for rats to acquire more than a weak lever-reinforcement association at best. Although this attentional-plus-associative approach may provide a plausible analysis of positive transfer, it is not easily applied to the negative transfer found in Experiment 3. Furthermore, the magazine entry data from Experiment 4 suggest that the absence of lever pressing by rats given the 10-sec-delay condition from the outset does not represent a failure to associate the lever with food. It is possible, however, that if a longer delay of reinforcement had been used, the role of an attentional process might have become more important.

As noted in the introduction, positive transfer from short to long ISIs has been reported in some previous experiments using classical conditioning procedures, notably those using the rabbit nictitating membrane preparation. One suggested analysis of this transfer is based on competition between CS and context, whereby slow acquisition of a conditioned response to a CS with a long ISI is seen as resulting from competing conditioning of contextual stimuli (Kehoe \& Holt, 1984; see also Dickinson, 1980; Westbrook \& Homewood, 1982). Applying this analysis to the present results implies that adding a procedure designed to extinguish context conditioning might lead to more rapid acquisition when reinforcement is delayed. However, the blocked control condition used in Experiment 3 would seem to rule out a context competition account of the negative transfer found in that experiment, since a high rate of lever pressing was recorded there during Stage 2 conditioning, despite prior training with noncontingent reinforcement in Stage 1. As noted above, introduction of a 10 -sec delay did not detectably retard acquisition of a lever-sucrose association, as measured by the development of magazine entries; thus, the role of context competition might also become more apparent for delays of reinforcement greater than $10 \mathrm{sec}$.

In conclusion, the simplest analysis that encompasses the results of Experiments 2, 3, and 4 is that inserting a 10-sec delay between lever retraction and responseindependent reinforcement leads to the development and persistence of behavior other than lever pressing. The results of Experiment 4 suggest that this behavior may take the form of goal tracking. Thus, positive transfer of sign tracking can be viewed as resulting from the nondevelopment of goal tracking when reinforcement is immediate or when - as in Experiment 3-blocked exposure to lever insertions and reinforcer deliveries is given. Whether the absence of competing behavior is the only factor responsible for such easy-to-hard transfer remains an open question.

\section{AUTHOR NOTE}

Experiments 1 and 2A were reported in D.S.J.C.'s unpublished honors thesis at the University of Sydney (Costa, 2004). Experiments 2B, 3, and 4 were partially supported by an Australian Research Council grant to R.A.B. and by an Australian Postgraduate Award to D.S.J.C. We thank Justin Harris and David Lieberman for their helpful comments on an earlier version of this article. Correspondence relating to this article may be sent to D. Costa, School of Psychology (A18), University of Sydney, Sydney, NSW 2006, Australia (e-mail: danielc@psych.usyd.edu.au).

\section{REFERENCES}

BoAkes, R. A. (1977). Performance on learning to associate a stimulus with positive reinforcement. In H. Davis \& H. M. B. Hurwitz (Eds.), Operant-Pavlovian interactions (pp. 67-101). Hillsdale, NJ: Erlbaum.

Bonardi, C., \& ONG, S. Y. (2003). Learned irrelevance: A contemporary overview. Quarterly Journal of Experimental Psychology, 56B, 80-89.

Brown, B. L., Hemmes, N. S., Cabeza de Vaca, S., \& Pagano, C. 
(1993). Sign and goal tracking during delay and trace autoshaping in pigeons. Animal Learning \& Behavior, 21, 360-368.

CosTA, D. S. J. (2004). The momentum effect: Maintenance of responding when reinforcement is delayed. Unpublished honors thesis, School of Psychology, University of Sydney.

Dickinson, A. (1980). Contemporary animal learning theory. Cambridge: Cambridge University Press.

Dickinson, A., WATt, A., \& GRIFFiths, W. J. (1992). Free-operant acquisition with delayed reinforcement. Quarterly Journal of Experimental Psychology, 45B, 241-258.

HARKER, G. S. (1956). Delay of reward and performance of an instrumental response. Journal of Experimental Psychology, 51, 303-310.

Holland, P. C. (1977). Conditioned stimulus as a determinant of the form of the Pavlovian conditioned response. Journal of Experimental Psychology: Animal Behavior Processes, 3, 77-104.

Kehoe, E. J., \& Holt, P. E. (1984). Transfer across CS-US intervals and sensory modalities in classical conditioning of the rabbit. Animal Learning \& Behavior, 12, 122-128.

LAWRENCE, D. H. (1952). The transfer of discrimination along a continuum. Journal of Comparative \& Physiological Psychology, 45 511-516.

Lieberman, D. A., McIntosh, D. C., \& Thomas, G. V. (1979). Learning when reward is delayed: A marking hypothesis. Journal of Experimental Psychology: Animal Behavior Processes, 5, 224-242.

Lieberman, D. A., \& Thomas, G. V. (1986). Marking, memory, and superstition in the pigeon. Quarterly Journal of Experimental Psychology, 38B, 449-459.

LoGAN, F. A. (1952). The role of delay of reinforcement in determining reaction potential. Journal of Experimental Psychology, 43 393-399.

Mackintosh, N. J. (1974). The psychology of animal learning. London: Academic Press.

MACKInTOSH, N. J. (1975). A theory of attention: Variations in the associability of stimuli with reinforcement. Psychological Review, 82 276-298.

Mackintosh, N. J., \& LitTLe, L. (1970). An analysis of transfer along a continuum. Canadian Journal of Psychology, 24, 362-369.

McGreevy, P., \& BoAKes, R. A. (2007). Carrots and sticks: Principles of animal training. Cambridge: Cambridge University Press.

Messing, R. B., Kleven, M. S., \& Sparber, S. B. (1986). Delaying reinforcement in an autoshaping task generates adjunctive and superstitious behaviors. Behavioural Processes, 13, 327-338.

Midgley, M., LeA, S. E. G., \& Kirby, R. M. (1989). Algorithmic shaping and misbehavior in the acquisition of token deposit by rats. Journal of the Experimental Analysis of Behavior, 52, 27-40.

Myer, J. S., \& Hull, J. H. (1974). Autoshaping and instrumental learning in the rat. Journal of Comparative \& Physiological Psychology, 86, 724-729.
Nevin, J. A., \& Grace, R. C. (2000). Behavioral momentum and the Law of Effect. Behavioral \& Brain Sciences, 23, 73-130.

Pavlov, I. P. (1927). Conditioned reflexes: An investigation of the physiological activity of the cerebral cortex (V. Anrep, Trans.). London: Oxford University Press.

Pear, J. J., \& Legris, J. A. (1987). Shaping by automated tracking of an arbitrary operant response. Journal of the Experimental Analysis of Behavior, 47, 241-247.

Perin, C. T. (1943). A quantitative investigation of the delay of reinforcement gradient. Journal of Experimental Psychology, 32, 37-52.

Prokasy, W. F., Ebel, H. C., \& Thompson, D. D. (1963). Response shaping at long interstimulus intervals in classical eyelid conditioning. Journal of Experimental Psychology, 66, 138-141.

Prokasy, W. F., \& PAPSDORF, J. D. (1965). Effects of increasing the interstimulus interval during classical conditioning of the albino rabbit Journal of Comparative \& Physiological Psychology, 60, 249-252.

Reilly, S., Schachtman, T. R., \& Reid, P. (1996). Signaled delay of reinforcement: Effects of postconditioning manipulation of context associative strength on instrumental performance. Learning \& Motivation, 27, 451-463.

RESCORLA, R. A. (1989). Redundant treatments of neutral and excitatory stimuli in autoshaping. Journal of Experimental Psychology: Animal Behavior Processes, 15, 212-223.

Rescorla, R. A., Durlach, P. J., \& Grau, J. W. (1985). Contextual learning in Pavlovian conditioning. In P. D. Balsam \& A. Tomie (Eds.), Context and learning (pp. 23-56). Hillsdale, NJ: Erlbaum.

Scahill, V. L., \& Mackintosh, N. J. (2004). The easy to hard effect and perceptual learning in flavor aversion conditioning. Journal of Experimental Psychology: Animal Behavior Processes, 30, 93-103.

Schmidt, R. A., \& BJork, R. A. (1992). New conceptualizations of practice: Common principles in three paradigms suggest new concepts for training. Psychological Science, 3, 207-217.

Stiers, M., \& Silberberg, A. (1974). Lever-contact responses in rats: Automaintenance with and without negative response-reinforcer dependency. Journal of the Experimental Analysis of Behavior, 22, 497-506.

Thorndike, E. L. (1911). Animal intelligence: Experimental studies. New York: Macmillan.

WestBRoOK, R. F., \& Homewood, J. (1982). The effects of a flavourtoxicosis pairing upon long-delay, flavour aversion learning. Quarterly Journal of Experimental Psychology, 34B, 59-75.

Williams, B. A. (1999). Associative competition in operant conditioning: Blocking the response-reinforcer association. Psychonomic Bulletin \& Review, 6, 618-623.

(Manuscript received September 6, 2006; revision accepted for publication December 12, 2006.) 